\title{
Solving the Two-Stage Cutting Stock Problem
}

\author{
ROBERT W HAESSLER
}

The University of Michigan, USA

(Receired July. 1978; in retised form Octoher 1978)

\begin{abstract}
This paper describes an important class of cutting stock problems not previously discussed in the literature. The problem is one of determining the patterns to be used in a two-stage cutting process with restrictions imposed on the locations of cuts in the first stage. A particularly difficult version of this problem from the plastic film industry is presented and solved.
\end{abstract}

\section{INTRODUCTION}

OVER THE past 20 years, a substantial amount of work has been done on one and two-dimensional cutting stock problems. A number of early authors $[1,6-8]$ recognized that the roll trim problem in the paper industry could be formulated as a linear programming model. The pioneering computational work by Gilmore and Gomory $[2,3]$ on delayed pattern generation techniques made it practical to find linear programming solutions to large onedimensional problems. Follow-up research by Pierce [9] focused on the use of this algorithm in the paper industry to solve the roll trim problem. Gilmore and Gomory [4] subsequently extended their work to higher dimensional problems such as the cutting of rectangles from rectangles, a common problem in the glass industry. They overcame the difficulty of generating patterns in higher dimensions by limiting themselves to patterns with guillotine cuts (once a cut is begun it must be continued through to the other side of the material in a straight line). As a result the problem of generating a higher dimensional cutting pattern can be handled by sequentially solving a number of one-dimensional problems. Their multi-stage approach in the two-dimensional case focuses first on cutting patterns for strips of known width and length and then on ways these strips can be combined to form twodimensional patterns.
In a number of industries, such as paper and film, there is an important class of problems that falls in between the well known one and two-dimensional problems. These are referred to here as two-stage cutting stock problems because the material is processed through two distinct cutting operations. It differs from the multi-stage cutting problems discussed by Gilmore and Gomory because the first and second stage cuts are parallel to one another. This problem has not been discussed in the literature even though it occurs in a number of industries. This two-stage cutting may be necessary because of the nature of the production process and the type of equipment available or to give flexibility so that orders that vary in some characteristic other than width can be combined across the width of the primary production process. Regardless of the reason, production rolls are first slit into master roll widths at the first stage and the master roll widths are then slit to the ordered sizes at the second stage. Limitations in the master roll sizes that can be processed at the second stage make it necessary to impose additional restrictions on the pattern generating process relative to the well known one-dimensional roll trim problem.

\section{GENERAL PROBLEM CONSIDERATIONS}

Consider the production process sketched in 


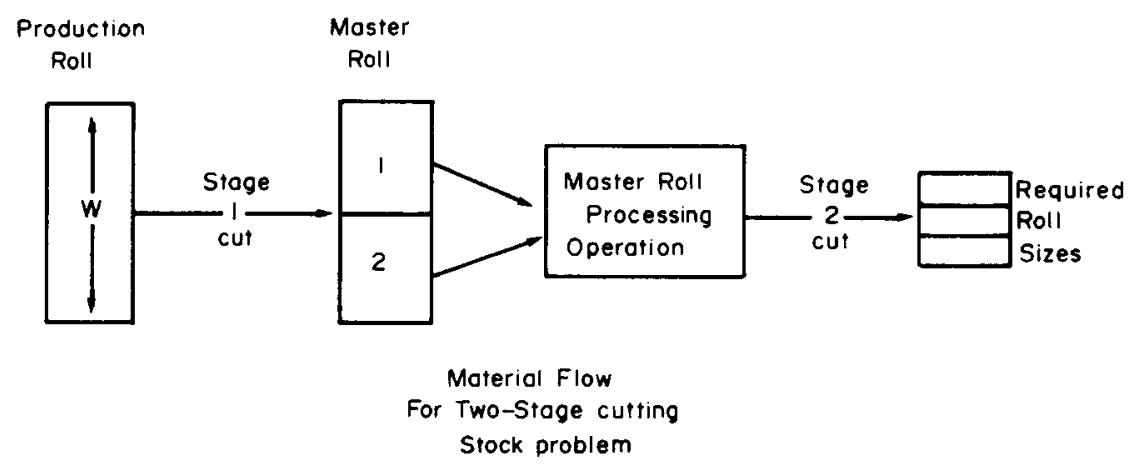

FIG. 1. Material flow for two-stage cutting stock problem.

Fig. 1. Let $W$ be the width of production rolls available to fill a known set of order requirements for rolls of some product. In the first cutting operation the production rolls are cut to obtain master rolls. After some additional processing these master rolls are cut to obtain the required roll sizes. These downstream operations place limits on the widths of the master rolls to be cut at the first stage. The problem is to specify how to cut the production rolls and resulting master rolls to meet the order requirements at minimum cost. If the production rolls could be cut in a single operation to obtain the required roll sizes, this would be a one-dimensional trim loss minimization problem. It is the size limitations imposed by the master roll processing operation as well as the costs incurred at this operation that make this problem unique.

Any cutting stock problem can be formulated as a trim loss minimization problem as follows:

$$
\begin{aligned}
\min \quad \sum_{p} x_{p} & \\
\sum_{p} A_{i p} x_{p} & =R_{i} \quad \text { all } i \\
x_{p} & \geq 0
\end{aligned}
$$

where

$x_{p}$ is the usage of pattern $p$.

$A_{i p}$ is the number of times size $i$ appears in pattern $p$,

and

$R_{i}$ is a measure of the required quantity for size $i$.

The differences in cutting stock problems can be seen only by defining what constitutes a feasible cutting pattern. In the one-dimensional problem with order sizes $W_{i}$ for $i=1, \ldots, m$, solved by Gilmore and Gomory, a cutting pattern is a set of values $A_{i p}$ for $i=1, \ldots, \ldots$ that satisfy the following conditions

$$
\begin{aligned}
\Sigma_{i} A_{i p} W_{i} & \leqslant W \\
A_{i p} & \geq 0, \quad \text { integer. }
\end{aligned}
$$

In the two-stage cutting problem considered here the $A_{i p}$ must satisfy (4) and (5) but also must be able to be partitioned into elements $A_{i p k}$ which satisfy the following conditions

$$
\begin{aligned}
A_{i p} & =\Sigma_{k} A_{i p k} \text { for all } i . p \\
\Sigma_{i} A_{i p k} W_{i} & \leq W M_{k} \text { for all } k \\
A_{i p k} & \geq 0, \quad \text { integer }
\end{aligned}
$$

where

$A_{i p k}$ is the number of times sizes $i$ appears in master roll $k$ for pattern $p$ and

$W M_{k}$ is the maximum width of master roll $k$.

In short, each pattern for the full width of the production roll can be thought of as defining a cutting stock problem with the master roll sizes defining the stock sizes available. This embedded cutting stock problem must have a feasible solution in order for the cutting pattern to be admissible. For example suppose that $W$, the maximum production width, is 200 inches and that each production roll must be slit into two master rolls neither of which can exceed 110 inches. A pattern containing eight 25 -inch rolls is satisfactory because two master rolls each containing four 25 -inch rolls can be cut. However, a pattern containing seven 28-inch rolls would not be acceptable if only two master rolls are to be cut because four times 28 is more than 110 . If more than two master rolls can be cut, this pattern could be 
used. However, the cost of processing three master rolls may make this pattern less desirable than one with the same trim loss that requires only two master rolls.

In addition to the above pattern restrictions, there may be additional differences relative to the one-dimensional roll trim problem. In some cases the first cutting operation may be done in such a way that the master rolls that are made side by side need not be the same diameter. This permits the possibility of combining orders of different diameters in the same pattern. In other cases, the master rolls may eventually be processed into sheets and therefore there may be some flexibility in determining the diameter of the master rolls and the diameters used may differ from pattern to pattern. Under these circumstances the second dimension of the order (i.e. roll diameter) does come into play and takes the problem beyond the one-dimensional problem. However, it is not really a two-dimensional problem because the length dimension of the stock size is not fixed. The handling of these considerations will differ from application to application. This will be demonstrated more fully in the film slitting problem described below.

\section{FILM SLITTING PROBLEM}

A particularly interesting example of a twostage cutting stock problem is found in the plastic film industry. In this case the film is slit at the end of the extruder on which it is formed. By slitting on the extruder the master rolls produced are independent of one another along the length of production, and therefore can be produced at different diameters. The master rolls are then taken immediately to the second stage slitters where they are slit to the ordered widths. The width capacity of the second stage slitters limits the master roll widths that can be produced.

Because the master roll diameters can be separately controlled, it is possible to consider problems that have mixed diameters or lineal footage requirements. This cannot be done in one-dimensional trim problems. The only requirement is that the orders to be slit from any master roll must all be of the same diameter. This simply puts an additional restriction on the conditions a pattern must satisfy to be feasible. The price that must be paid to obtain this flexibility with master roll diameters is that the process must be shut down to change the slit point setting. This introduces a fixed charge that is incurred each time the master roll widths must be changed.

Note that it is possible to generate scrap along the length of production as well as across the width. Suppose that 100,000 feet of film is to be produced for one slit point setting on the extruder. On one side of the slit point it is possible to produce five master rolls each of which has a length of 20,000 feet. Suppose that on the other side of the slit point the master rolls required must have 16,000 feet of material. Only six of these can be produced and there will be 4,000 feet of material left over that must be scrapped or held in inventory until some use can be found for it.

To formulate this problem let

$$
\begin{aligned}
& W_{i} \quad \begin{array}{l}
\text { be the roll width order } i \\
L_{i}
\end{array} \begin{array}{l}
\text { be the lineal footage for each roll } \\
\text { for order } i
\end{array} \\
& R L_{i} \quad \begin{array}{l}
\text { be the lower bound on the rolls } \\
\text { required to satisfy order } i
\end{array} \\
& R U_{i} \quad \begin{array}{l}
\text { be the upper bound on the rolls } \\
\text { required to satisfy order } i
\end{array} \\
& W \quad \begin{array}{l}
\text { be the width of the extruder } \\
\text { be the maximum width for master } \\
\text { roll } k
\end{array} \\
& C_{1} \quad \begin{array}{l}
\text { be the value of a unit area of trim } \\
\text { loss }
\end{array} \\
& C_{2} \quad \begin{array}{l}
\text { be the cost of shutting down the } \\
\text { extruder to change the slit point set- } \\
\text { ting }
\end{array}
\end{aligned}
$$

The problem is to produce the order requirements within the tolerances specified, at a minimum combined cost of trim loss and downtime. The problem can be formulated mathematically as follows:

$$
\min C_{1}\left(W \Sigma_{j} X_{j}-\Sigma_{i} Y_{i} L_{i} W_{i}\right)+C_{2} \Sigma_{j} \delta\left(X_{j}\right)
$$

subject to

$$
\begin{gathered}
R L_{i} \leq Y_{i} \leq R U_{i} \text { for all } i, \\
Y_{i}=\Sigma_{j} \Sigma_{k} \Sigma_{p} A_{i j k p}\left[X_{j k_{p}} / L_{i}\right] \text { for all } i \\
X_{j} \geq \Sigma_{p} X_{j k p} \text { for all } j \text { and } k
\end{gathered}
$$

and

$$
X_{j}, X_{j k p} \geq 0
$$

where the remaining symbols are defined as follows: 
$Y_{i}$-number of rolls produced for order $i$.

$X_{j}$-number of feet of production for the $j$ th set of master roll widths. (A set of master roll widths is determined by the slit point setting at the extruder.

$\delta\left(X_{j}\right)=1$ if $X_{j}>0,0$ otherwise

$A_{i j k p}$-number of times order $i$ appears in pattern $p$ for master roll $k$ and slit point setting $j$.

$X_{j k p}$-number of feet of production of master roll $k$ processed according to pattern $p$ for slit point setting $j$.

$\left[X_{j k p} / L_{i}\right]$-largest integer less than or equal to $X_{j k p} / L_{i}$.

To aid in the discussion, let $Z_{j k}$ be the width of master roll $k$ for the $j$ th set of master roll sizes. It follows that:

$$
\begin{aligned}
\Sigma_{k} Z_{j k} & =W \text { for all } j . \\
Z_{j k} & \leq W M_{k} \text { for all } j \text { and } k, \\
\Sigma_{i} A_{i j k p} W_{i} & \leq Z_{j k} \text { for all } j, k \text { and } p,
\end{aligned}
$$

and

$$
A_{i j k p} \geq 0, \quad \text { integer }
$$

This formulation contains some extreme combinatorial aspects and is far beyond the capability of currently available optimization procedures. Therefore attention is directed toward the problem of finding 'good' feasible solutions. The primary objective is to develop a procedure that generates better solutions than are obtainable by currently available manual methods. Although the mathematical model can not be solved for an optimal solution, it does provide a basis for testing solution feasibility and evaluating solution quality regardless of how the solution is generated.

\section{SOLUTION PROCEDURE}

A computer program that employs a number of heuristics in conjunction with a linear programming algorithm was developed to solve this problem for a film producer [5]. A detailed description of the solution procedure follows:

1. Compute the total lineal footage for each order.

$$
F_{i}=R_{i} L_{i}
$$

This then becomes the required quantity in a linear programming problem.

$$
\begin{aligned}
\operatorname{minimize} \quad \Sigma_{p} X_{p} \\
\text { subject to } \quad \Sigma_{p} A_{i p} X_{p}=F_{i} \text { for } i=1 \ldots m, \\
X_{p} \geq 0
\end{aligned}
$$

where

$X_{p}$-number of feet of production to process using pattern $p$.

$A_{i p}$-number of times size $i$ appears in pattern $p$.

2. Solve the problem given above using a Gilmore-Gomory $[1,2]$ type linear programming algorithm. This algorithm is based on the principle of delayed pattern generation. All possible patterns are not generated at the outset. Patterns that will improve the solution are generated as needed by solving an associated problem to find that pattern that will give the greatest incremental improvement. This pattern generation process is restricted such that only patterns that can be partitioned to meet the slitter width and length restrictions are considered. This insures solution feasibility across the width of the extruder. A feasible cutting pattern in a classical roll slitting problem can be defined by elements $A_{i p}$ such that:

$$
\begin{aligned}
\Sigma_{i} A_{i p} W_{i} & \leq W \\
A_{i p} & \geq 0, \text { integer }
\end{aligned}
$$

In. this case, the $A_{i p}$ must also be partitioned into elements $A_{i k p}$, the number of times size $i$ appears in master roll $k$ for pattern $p$. The following restrictions must be met:

$$
\begin{aligned}
\Sigma_{k} A_{i k p} & =A_{i p} \text { all } i \text { and } p \\
\Sigma_{i} A_{i k p} W_{i} & \leq W M_{k} \text { all } k
\end{aligned}
$$

In addition, because of slitter limitations, all the orders contained in a master roll pattern must have the same lineal footage.

The solution to the linear programming problem minimizes the trim loss and the requirements in terms of footage, $F_{i}$, for each order are met exactly. However, this solution is generally of no use because it usually will require a substantial amount of splicing to obtain rolls of the desired lineal footage. In most situations, splicing is not even allowed, therefore the pattern production quantities must be determined for each master roll pattern as multiples of the lineal footage of the 
involves finding the slit point settings and grouping the patterns that can be produced at the same setting.

3. Determine the number and location of the slit point settings for the patterns found in 2 . This can be done by computing the minimum and maximum possible width for the widest master roll in each pattern, finding the smallest of the maximums, and taking that as a slit point setting and assigning as many full width patterns as possible to that setting. The minimum and maximum widths will not be the same if $W>\Sigma_{i} A_{i p} W_{i}$, there is trim loss in the pattern. This procedure is repeated until all patterns are assigned to a setting. At this point $X_{p}$ becomes $X_{j p}$, where $j$ identifies the slit point setting. This procedure will give the minimum number of settings when there is only one slit point at the extruder. When there is more than one slit point, the procedure must be applied to each slit point. It is no longer possible to guarantee that the minimum number will be found.

4. For each master roll width in each pattern, find a length that will yield an integer number of rolls. This is done by dividing $X_{j p}$ by $L_{i}$, the length of the orders in that master roll and rounding to the nearest integer. Now $X_{j p}$ becomes $X_{j k p}$ where $X_{j k p}$ is an integer multiple of the order length that is being made in that master roll pattern.

5. Compute the total length for each master roll setting.

$$
X_{j}=\max _{k}\left(\Sigma_{p} X_{j k p}\right)
$$

and compute the number of each size roll that is being produced.

At this point, there are three potential difficulties with the solution.

(a) The amount produced for an order may be less than the lower bound of the requirement as a result of step 4 .

(b) There may be excessive trim loss along the length for a given master roll width. The unused portion of master roll $k$ for slit point setting $j$ is $X_{j}-\Sigma_{p} X_{j k p}$.

(c) The solution may involve too many slit point settings.

These three potential problems are handled as follows:
6. Increase the production length of the first master roll pattern found that contains a size for which the production quantity is insufficient to meet the lower bound, provided that it does not cause some other item to be produced at a level above its upper bound. This process is repeated until either all the items fall within the production limits or until no further progress can be made. Each time the length of a master roll pattern is increased, the values of $X_{j}$ and $X_{j k p}$ must be changed accordingly.

7. Check each slit point setting to determine if there is a significant length of unused material for any master roll. If there is a loss for master roll $t$, do the following in the order given.

(a) Reduce the usage $X_{j k p}$ of patterns for master rolls $k \neq t$ to reduce the $\Sigma_{p} X_{j k p}$ and thereby be able to reduce $X_{j}$. This reduction is done only if the order requirements are not violated.

(b) Increase the usage $X_{j k p}$ of some pattern for master roll $k=t$ to make use of the unutilized material. Once again this is done only if the upper bound for each order is not violated.

At this point a feasible solution should have been found, and the only question remaining is whether it is economically desirable to reduce the number of slit point settings. Reducing the number of slit point settings will generally increase the trim loss (it cannot reduce the trim loss except for differences due to rounding). The issue is whether or not the increase in trim loss is less than the gain in production obtained by eliminating a slit point change.

8. Determine the slit point setting which has the lowest usage $X_{j}$. Eliminate it from the set of slit point settings than can be used during the next solution to the problem. This can be done by specifying a set of maximum master roll widths $Z_{j k}$ such that:

$$
\Sigma_{k} Z_{j k}=W \text { for all } j
$$

The entire problem is then resolved. The only difference is that step 3 can be eliminated. The patterns are assigned to one of the allowed slit point settings at the time they are generated. This process repeats until the increased cost of trim loss due to reducing the number of 
orders scheduled. The first step in this process master roll settings more than offsets the decrease in the cost of downtime made possible by reducing the number of master roll settings.

The tradeoff between trim loss and downtime is handled by specifying the number of feet of production lost while changing the master roll settings. This is multiplied by $W$ to find the area of production lost. This is then added to the actual trim loss to find the effective loss of a solution.

\section{EXAMPLE}

An example problem is given in Table 1. The extruder in this example is capable of producing film with a maximum usable width of 124 inches. The two second stage slitters that process the master rolls have maximum widths of 76 and 55 inches. This means that as the film comes off the extruder it must be slit exactly once at a point that is within 76 inches of one side and 55 inches of the other so the resulting master rolls can be processed through the two second stage slitters. It is not possible to produce two 62-inch width master rolls because they would both have to go the larger slitter and the slitter processing speed is not sufficient to keep up with the extruder under these conditions.

The solution to this problem is presented in Table 2. The lost production of a slit point change was specified as 30,000 feet. The result-

Table 1. Sample PRoblem

Production data

Extruder width -124 inches

Number of slit points-1

Width of slitter $1-76$ inches

Width of slitter 2-55 inches

Roll order requirements

\begin{tabular}{rcc}
\hline Quantity & Width & Lineal footage/roll \\
\hline 7 & 55.0000 & 20000. \\
3 & 39.0000 & 20000. \\
4 & 36.5000 & 35000 \\
1 & 36.0313 & 10000 \\
36 & 26.0000 & 10000 \\
270 & 24.5000 & 15000. \\
4 & 21.0000 & 15400. \\
4 & 21.0000 & 15400. \\
3 & 19.0000 & 20000. \\
7 & 18.2500 & 20000. \\
4 & 17.8750 & 20000. \\
\hline
\end{tabular}

Table 2. Problem SOlution

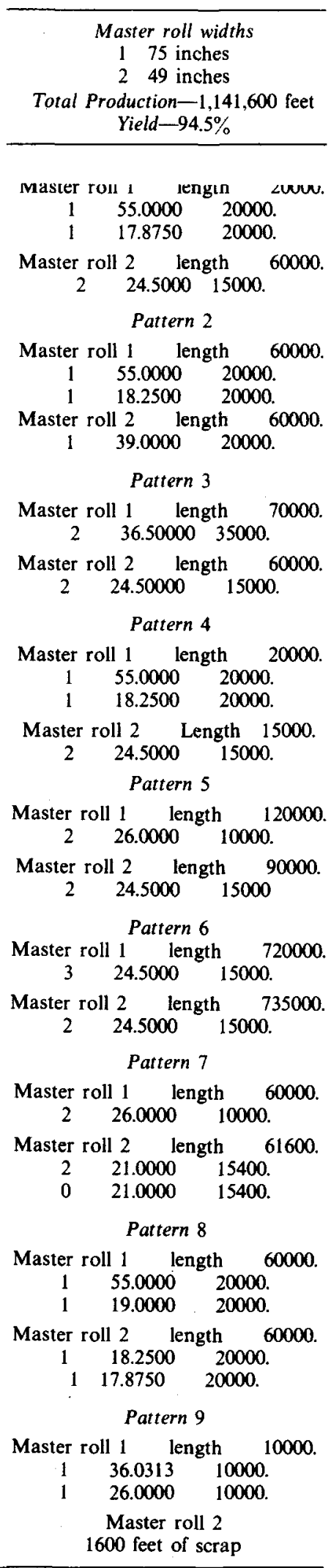


ing solution has only one slit point setting and an overall yield of $94.5 \%$ on scheduled production of 1,141,600 feet. There are 9 master roll patterns for the 76 inch slitter and 8 for the 55 inch slitter. There are 1,600 feet of unutilized material on the master rolls going to the first slitter. This is treated as scrap. The master roll patterns are given in pairs to indicate the manner in which they were generated. The differences in usage for corresponding master roll patterns indicates the amount of adjusting that is done to meet the order requirements and avoid leaving unutilized material. The only deviations from the ordered quantities are the following overruns, all within the $10 \%$ deviation allowed.

$$
\begin{array}{ll}
1 & 55^{\prime \prime} \\
1 & 26^{\prime \prime} \\
2 & 24.5^{\prime \prime}
\end{array}
$$

The solution time for this problem was less than two seconds on an Amdahl 470V/6.

\section{SUMMARY}

This paper has described an important class of cutting stock problems that falls between the well known one and two-dimensional cases. In the example given, the two-stage cutting operation was installed in order to provide flexibility so that orders for rolls of different diameters could be combined across the width of the extruder. If a two-stage cutting operation was not used, the orders in Table 1 would have to be grouped by lineal footage and each group would then represent a separate one-dimensional cutting stock problem. It should be clear that subgrouping the orders in this way would severely restrict the patterns that could be used. Regardless of their reasons for existence. two-stage cutting stock problems do represent an important class of cutting stock problems to be solved. The example presented here. demonstrates how a modification to the Gilmore-Gomory one dimensional algorithm can be used to obtain high quality solutions to these problems.

\section{REFERENCES}

1. Eisemann K (1957) The trim problem. Mgmt Sci. 3. 279-284.

2. GILMORE PC \& GOMORY RE (1961) A linear programming approach to the cutting-stock problem. Ops Res. 9. 849-859.

3. Gilmore PC \& Gomory RE (1963) A linear programming approach to the cutting-stock problem - part II. Ops Res. 11, 863-888.

4. GILMORE PC \& GOMOR Y RE (1963) Multistage cuttingstock problems of two and more dimensions. Ops Re's. 11, $94-120$

5. Haessler RW (1976) A procedure for solving the film cutting-stock problem. ORSA-TIMS Meeting. Philadelphia.

6. Kantorovich LV (1960) Mathematical methods of organizing and planning production. Mymt Sci. 6, $366-422$.

7. Metzger RW (1958) Stock shiting. Elementary Mathematical Programming. Ch. 8. John Wiley. New York.

8. Paul AE \& Walter JR (1955) The trim problem: an application of linear programming to the manufacture of newsprint. Paper presented at annual meeting of Econometrica Society, Montreal (1954). Econometrica 23(3), 336.

9. PIERCE JF (1964) Some Large Scale Production Scheduling Problems in the Paper Industry. Prentice- Hall. Englewood Cliffs, New Jersey.

ADDRESS FOR CORRESPONDENCE: Rohert $W$ Haessler Esq. Associate Professor of Policy and Control. The Unitersity of Michigan. Graduate School of Business Administration. Michigan 48109. USA. 